\title{
Aspirin, clopidogrel and prasugrel monotherapy in patients with type 2 diabetes mellitus: a double-blind randomised controlled trial of the effects on thrombotic markers and microRNA levels
}

William A. E. Parker ${ }^{1}$ (1), Christian Schulte ${ }^{2,3}$, Temo Barwari ${ }^{2}$, Fladia Phoenix ${ }^{4}$, Sam M. Pearson ${ }^{4}$, Manuel Mayr ${ }^{2}$, Peter J. Grant ${ }^{4}$, Robert F. Storey ${ }^{1}$ and Ramzi A. Ajjan ${ }^{*^{*}}$

\begin{abstract}
Background: Despite increased atherothrombotic risk in type 2 diabetes mellitus, (T2DM) the best preventative antithrombotic strategy remains undetermined. We defined the effects of three antiplatelet agents on functional readout and biomarker kinetics in platelet activation and coagulation in patients with T2DM.

Materials and methods: 56 patients with T2DM were randomised to antiplatelet monotherapy with aspirin $75 \mathrm{mg}$ once daily (OD), clopidogrel $75 \mathrm{mg}$ OD or prasugrel $10 \mathrm{mg}$ OD during three periods of a crossover study. Platelet aggregation (PA) was determined by light-transmittance aggregometry and P-selectin expression by flow cytometry. Markers of fibrin clot dynamics, inflammation and coagulation were measured. Plasma levels of 14 miRNA were assessed by quantitative polymerase chain reactions.
\end{abstract}

Results: Of the 56 patients, 24 (43\%) were receiving aspirin for primary prevention of ischaemic events and 32 (57\%) for secondary prevention. Prasugrel was the strongest inhibitor of ADP-induced PA (mean \pm SD maximum response to $20 \mu \mathrm{mol} / \mathrm{L}$ ADP $77.6 \pm 8.4 \%$ [aspirin] vs. $57.7 \pm 17.6 \%$ [clopidogrel] vs. $34.1 \pm 14.1 \%$ [prasugrel], $\mathrm{p}<0.001$ ), P-selectin expression (30 $\mu \mathrm{mol} / \mathrm{L}$ ADP; $45.1 \pm 21.4 \%$ vs. $27.1 \pm 19.0 \%$ vs. $14.1 \pm 14.9 \%, \mathrm{p}<0.001)$ and collagen-induced PA $(2 \mu \mathrm{g} /$ $\mathrm{mL} ; 62.1 \pm 19.4 \%$ vs. $72.3 \pm 18.2 \%$ vs. $60.2 \pm 18.5 \%, p<0.001$ ). Fibrin clot dynamics and levels of coagulation and inflammatory proteins were similar. Lower levels of miR-24 ( $p=0.004)$, miR-191 $(p=0.019)$, miR-197 $(p=0.009)$ and miR-223 ( $p=0.014$ ) were demonstrated during prasugrel-therapy vs. aspirin. Circulating miR-197 was lower in those cardiovascular disease during therapy with aspirin $(p=0.039)$ or prasugrel $(p=0.0083)$.

Conclusions: Prasugrel monotherapy in T2DM provided potent platelet inhibition and reduced levels of a number of platelet-associated miRNAs. miR-197 is a potential marker of cardiovascular disease in this population. Clinical outcome studies investigating prasugrel monotherapy are warranted in individuals with T2DM.

Trial registration EudraCT, 2009-011907-22. Registered 15 March 2010, https://www.clinicaltrialsregister.eu/ctr-search/ trial/2009-011907-22/GB.

Keywords: Aspirin, Diabetes mellitus, Micro RNA, P2Y ${ }_{12}$ inhibitors, Platelet inhibition

*Correspondence: R.Ajjan@leeds.ac.uk

${ }^{4}$ Leeds Institute of Cardiovascular and Metabolic Medicine, University

of Leeds, Leeds, UK

Full list of author information is available at the end of the article

c) The Author(s) 2020. This article is licensed under a Creative Commons Attribution 4.0 International License, which permits use, sharing, adaptation, distribution and reproduction in any medium or format, as long as you give appropriate credit to the original author(s) and the source, provide a link to the Creative Commons licence, and indicate if changes were made. The images or other third party material in this article are included in the article's Creative Commons licence, unless indicated otherwise in a credit line to the material. If material is not included in the article's Creative Commons licence and your intended use is not permitted by statutory regulation or exceeds the permitted use, you will need to obtain permission directly from the copyright holder. To view a copy of this licence, visit http://creativecommons.org/licenses/by/4.0/. The Creative Commons Public Domain Dedication waiver (http://creativecommons.org/publicdomain/zero/1.0/) applies to the data made available in this article, unless otherwise stated in a 


\section{Background}

Thrombotic events are associated with a large burden of morbidity and mortality in the general population [1], with an even higher risk in patients with type 2 diabetes mellitus (T2DM) [2]. Enhanced platelet activation and altered fibrin clot properties are central pathological processes in the development of thrombosis in T2DM, thus increasing the risk of cardiovascular events and contributing to adverse clinical outcome following vascular ischaemia [3-5].

Antiplatelet drugs for the treatment and prevention of atherothrombosis have largely focussed on two pathways of platelet activation: thromboxane $\mathrm{A}_{2}$ generation, which is blocked by the irreversible cyclo-oxygenase inhibitor aspirin, and adenosine diphosphate- (ADP-) induced amplification of platelet activation via the $\mathrm{P} \mathrm{Y}_{12}$ receptor, which is irreversibly inhibited by thienopyridines such as clopidogrel and prasugrel [6]. In combination with aspirin, thienopyridines reduce the risk of thrombotic events after acute coronary syndrome (ACS) [7], but the protective effects can vary according to the agent used and the population studied. Following coronary ischaemia requiring percutaneous intervention, prasugrel has shown enhanced vascular protective properties compared with clopidogrel in T2DM patients without an increase in bleeding risk, in contrast to individuals without T2DM $[8,9]$.

In the clopidogrel vs aspirin in patients at risk of ischemic events (CAPRIE) study, clopidogrel, used as single antiplatelet therapy (SAPT) showed better protection against vascular ischaemia compared with aspirin monotherapy in patients with T2DM [10], an effect that was even more pronounced in insulin-treated subjects [11]. In contrast to clopidogrel, prasugrel as SAPT has not been well studied and data on patients with T2DM are scarce.

Studies of aspirin for the primary prevention of cardiovascular disease in patients T2DM have been disappointing, with little or no reduction in vascular ischaemic events at the expense of a significant increase in bleeding risk [12-16]. Similarly, the pharmacokinetics and clinical efficacy of clopidogrel, a pro-drug, display significant variability between individuals due to differences in activity of cytochrome P450 2C19 [17]. Prasugrel, whilst also a pro-drug, is activated by a different, more predictable metabolic pathway and therefore offers better inter-individual consistency of effect [18].

In addition to the effects on platelets, aspirin and $\mathrm{P} 2 \mathrm{Y}_{12}$ inhibitors may modulate the fibrin network $[19,20]$ and affect vascular inflammatory pathways [21, 22]. In order to assess a functional readout and to gain further mechanistic insight, platelet function tests have been used to assess the response to antiplatelet agents. Micro-ribonucleic acids (miRNAs) are emerging as an adjunct to our understanding and assessment of platelet function [23-25]. From a translational point of view, detection of a variety of miRNAs has been linked with clinical outcomes including ischaemic heart disease [26].

Prasugrel monotherapy in individuals with T2DM may offer superior anti-thrombotic properties compared with aspirin or clopidogrel. The aim of this study was to comprehensively characterise and compare the effects of the three drugs on platelet function, fibrin network characteristics, inflammation and expression of miRNAs in a cohort of T2DM patients.

\section{Methods \\ Study design}

We performed a single-centre, double-blind, crossover, randomised controlled trial of patients with a confirmed diagnosis of T2DM. Patients were eligible to participate if they were aged 18-75 years, already on treatment with aspirin $75 \mathrm{mg}$ once-daily (OD) and able to give informed consent. Eligible participants receiving aspirin $75 \mathrm{mg}$ OD were randomised 1:1 to one of two medication sequences (Fig. 1). All patients continued aspirin $75 \mathrm{mg}$ OD for an initial lead-in period of 14 days. One half then received clopidogrel $75 \mathrm{mg}$ OD for 28 days then prasugrel $10 \mathrm{mg}$ OD for 28 days, whilst the other half received prasugrel $10 \mathrm{mg}$ OD for 28 days followed by clopidogrel $75 \mathrm{mg}$ OD

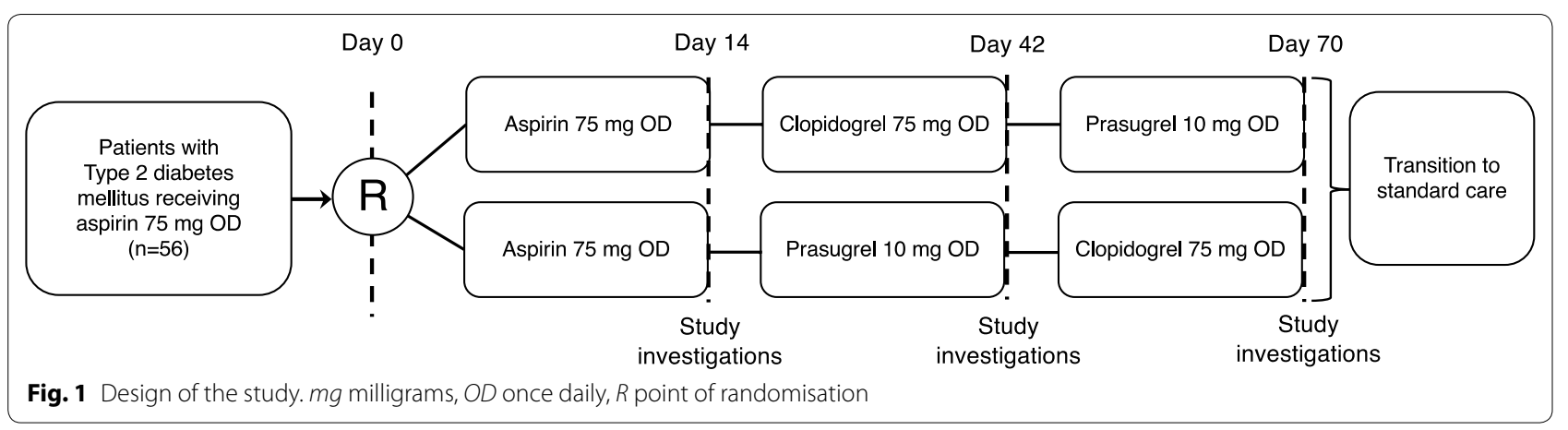


for 28 days. Aspirin was discontinued after study completion if it was deemed clinically unnecessary. Randomisation was performed by shuffled sealed opaque envelopes prepared by a member of pharmacy staff. Both patients and investigators were blind to treatment allocation until all study data was collected. A range of pharmacodynamic measurements were made at the end of each treatment period. The study was approved by the United Kingdom National Health Service Research Ethics Service (reference 09/H1307/110). Written consent was obtained from participants before any study activities took place.

Exclusion criteria included: any type of diabetes other than T2DM; any coagulation disorder; neoplastic disease; history of ACS within 3 months of enrolment; history of stroke or transient ischaemic attack; history of deep venous thrombosis or pulmonary embolism; treatment with oral anticoagulant or non-steroidal antiinflammatory drugs; abnormal liver enzyme tests defined as alanine transferase > threefold upper limit of normal; any previous or current upper gastrointestinal pathology; weight < $60 \mathrm{~kg}$; and women of child-bearing age and refusing to use contraception.

\section{Blood samples}

Venous blood samples were collected by venepuncture, using syringe and $18 \mathrm{G}$ needle, and anticoagulated with trisodium citrate dihydrate $3.13 \%$. Platelet-rich plasma was prepared by centrifugation for $10 \mathrm{~min}$ at $200 \times g$ and platelet-poor plasma was prepared by further centrifugation for $10 \mathrm{~min}$ at $1500 \times \mathrm{g}$. All analyses described below were undertaken by individuals blinded to the sample details and type of antiplatelet treatment.

\section{Platelet aggregation}

Light transmittance aggregometry (LTA) was performed using $\operatorname{ADP}(1,2,5,10$ and $20 \mu \mathrm{mol} / \mathrm{L})$, arachidonic acid (AA, $1.0 \mathrm{mmol} / \mathrm{L})$ and collagen $(2$ and $16 \mu \mathrm{g} / \mathrm{mL})$ as agonists and a PAP-8 aggregometer (v2.0, Bio/Data Corporation, Horsham, PA, USA), as previously described [27]. Maximum (MA) and final (FA) aggregation responses at 6 min after agonist injection, adjusted for baseline, were recorded. Samples were assessed in duplicate, taking the mean value for analysis, and repeated if a discrepancy of $>10 \%$ was observed between the readings.

\section{Platelet P-selectin expression}

Surface expression of platelet P-selectin after stimulation with $0.3,1,3,10 \& 30 \mu \mathrm{mol} / \mathrm{L}$ ADP was quantified using flow cytometry and expressed as percentage positive events, as previously described [28].

\section{Fibrin clot turbidimetric analysis}

High-throughput turbidimetric analysis was performed as previously described and validated [29-32]. Briefly, citrated platelet-poor plasma samples were mixed with standard lysis and activation mixes to form acellular clots. Serial absorbance was measured using an automated plate reader during clot formation until lysis was achieved. Variables recorded were lag time, representing the period from the addition of clot activation mix to the start of clot formation (a measure of clotting tendency), final clot turbidity (maximum absorbance, a representation of fibre thickness and clot density), and lysis time (time from full clot formation to $50 \%$ lysis, a measure of fibrinolysis potential).

\section{Coagulation-associated proteins and inflammatory markers}

Levels of plasminogen activator inhibitor 1 (PAI1) were determined in citrated plasma using enzymelinked immunosorbent assay, as previously described [32]. Fibrinogen levels were determined by the clotting method of Clauss using a KC 10TM coagulometer (Henrich Amelung GmbH, Lemgo, Germany), as described elsewhere [33, 34]. Levels of C-reactive protein (CRP) and complement $\mathrm{C} 3$ were determined, as previously described [31].

\section{RNA isolation and miRNA quantification}

Quantification of miRNAs with known relevance to platelet function and cardiovascular disease (miRs 21, 24, 27b, 28, 93, 122, 126, 150, 191, 197, 223, 320, 451a and 486) was performed in platelet-poor plasma samples using reverse transcription quantitative polymerase chain reaction (RT-qPCR), as previously described [24, 26]. Total RNA was isolated using the miRNeasy Mini kit (Qiagen, Hilden, Germany). In brief, $100 \mu \mathrm{L}$ of plasma were combined with $694.75 \mu \mathrm{L}$ of QIAzol, $4 \mu \mathrm{L}$ of diluted Caenorhabditis elegans miR-39-3p (cel-miR-39) spike-in and $1.25 \mu \mathrm{L}$ carrier MS2. Following a brief incubation at ambient temperature, $140 \mu \mathrm{L}$ of chloroform were added and the solution was mixed vigorously. Samples were then centrifuged at 13,500 relative centrifugal force ( $\mathrm{rcf}$ ) for $15 \mathrm{~min}$ at $4{ }^{\circ} \mathrm{C}$. The upper aqueous phase was carefully transferred to a new tube and 1.5 volumes of ethanol were added. The samples were then applied directly to columns and washed according to the company's protocol. Total RNA was eluted with $35 \mu \mathrm{L}$ of nuclease-free water. A fixed volume of $3 \mu \mathrm{L}$ of the $35 \mu \mathrm{L}$ RNA eluate was used as input for reverse transcription (RT) reactions. MiRNAs were reverse-transcribed using Megaplex RT Primer Pools (Human Pool A version 2.1; Life Technologies, Darmstadt, Germany) and the TaqMan MicroRNA RT kit (Life Technologies, Darmstadt, 
Germany) according to the manufacturer's instructions. Templates were pre-amplified using Megaplex PreAmp Primers (Primers A version 2.1) and PreAmp Mastermix (Life Technologies) with 12 cycles of $95{ }^{\circ} \mathrm{C}$ for $15 \mathrm{~s}$ and $60{ }^{\circ} \mathrm{C}$ for $4 \mathrm{~min}$. Pre-amplification product was 72 times diluted and $2.25 \mu \mathrm{L}$ were combined with $0.25 \mu \mathrm{L}$ TaqMan microRNA assay $(20 \times)$ (Life Technologies) and $2.5 \mu \mathrm{L}$ TaqMan Universal PCR Master Mix No AmpErase UNG $(2 \times)$ to a final volume of $5 \mu \mathrm{L}$. RT-qPCR was performed on an Applied Biosystems Viia 7 thermocycler at $95{ }^{\circ} \mathrm{C}$ for $10 \mathrm{~min}$, followed by 40 cycles of $95{ }^{\circ} \mathrm{C}$ for $15 \mathrm{~s}$ and $60{ }^{\circ} \mathrm{C}$ for $1 \mathrm{~min}$. Clinical data were blinded to laboratory personnel.

Cel-miR-39 was used for normalisation and as a quality control. Quantification results were calibrated with pooled RNA from 50 samples. Quantification cycle (Cq) values $>32$ were considered to fall below the limit of detection. Relative quantification was performed with Microsoft Excel, version 15.32 for Mac using the $2^{(-\Delta \Delta \mathrm{Cq})}$ method [35].

\section{Statistical analysis}

The three treatments were compared by repeated measures ANOVA with Greenhouse-Geisser correction. The primary endpoints of the study were platelet aggregation responses to ADP, collagen and AA; platelet P-selectin expression and fibrin clot dynamics. Other analyses were exploratory. For variables with a significant difference $(\mathrm{p}<0.05)$ between the treatments, pairwise comparisons were performed using Bonferroni correction. SPSS statistics v25 (IBM software) was used for these analyses and graphical representations generated using GraphPad PRISM v7. Correlation and subgroup analyses were performed using RStudio v1.1.456: adjustment for multiple comparisons was not made for these as they were exploratory and intended for hypothesis generation.

A total of 56 patients were needed to detect a $10 \%$ difference in platelet aggregation response to various agonists comparing the different therapies at $\mathrm{p}<0.05$ and $90 \%$ power, based on the assumption that the common standard deviation of the response variable is $16 \%$. The study also had the power to detect a $7 \%$ difference in clot final turbidity based on the assumption that standard deviation for the response variable is $11 \%(\mathrm{p}<0.05,90 \%$ power).

\section{Results}

\section{Recruitment and participant characteristics}

Of 310 patients who were approached, 64 were enrolled and 56 completed the study (Additional file 1: Figure S1). Baseline characteristics are shown in Table 1.
Table 1 Baseline characteristics of participants completing the study

Participants $(n=56)$

\begin{tabular}{|c|c|}
\hline \multicolumn{2}{|l|}{ Sex } \\
\hline Female/male & $9 / 47$ \\
\hline \multicolumn{2}{|l|}{ Age (years) } \\
\hline Mean (range) & $60.7(46-73)$ \\
\hline Smoking $(\mathrm{Y} / \mathrm{N})$ & $11 / 45$ \\
\hline \multicolumn{2}{|l|}{ Blood pressure [mean \pm SD] } \\
\hline Systolic $(\mathrm{mmHg})$ & $132.0 \pm 16.32$ \\
\hline Diastolic $(\mathrm{mmHg})$ & $80.0 \pm 9.51$ \\
\hline \multicolumn{2}{|l|}{ Physical examination [mean $\pm \mathrm{SD}$ ] } \\
\hline Height (m) & $1.72 \pm 0.07$ \\
\hline Weight (kg) & $96.18 \pm 17.60$ \\
\hline BMl & $32.70 \pm 5.23$ \\
\hline \multicolumn{2}{|l|}{ Baseline blood tests [mean $\pm S D$ ] } \\
\hline $\mathrm{HbA} 1 \mathrm{c}(\mathrm{mmol} / \mathrm{mol})$ & $71.60 \pm 22.99$ \\
\hline Sodium (mmol/L) & $139.28 \pm 2.39$ \\
\hline Potassium (mmol/L) & $4.48 \pm 0.39$ \\
\hline Creatinine $(\mu \mathrm{mol} / \mathrm{L})$ & $83.13 \pm 20.66$ \\
\hline Urea $(\mathrm{mmol} / \mathrm{L})$ & $6.20 \pm 2.41$ \\
\hline $\mathrm{eGFR}\left(\mathrm{mL} / \mathrm{min} / 1.73 \mathrm{~m}^{2}\right)$ & $80.70 \pm 13.24$ \\
\hline Total cholesterol (mmol/L) & $3.79 \pm 0.77$ \\
\hline $\mathrm{LDL}(\mathrm{mmol} / \mathrm{L})$ & $1.89 \pm 0.49$ \\
\hline $\mathrm{HDL}(\mathrm{mmol} / \mathrm{L})$ & $1.08 \pm 0.26$ \\
\hline Triglycerides (mmol/L) & $2.06 \pm 2.01$ \\
\hline $\mathrm{FT}_{4}(\mathrm{pmol} / \mathrm{L})$ & $15.10 \pm 2.12$ \\
\hline TSH (mIU/L) & $2.00 \pm 1.12$ \\
\hline History of macrovascular disease & $32(57 \%)$ \\
\hline \multicolumn{2}{|l|}{ Microvascular complications } \\
\hline Retinopathy & $23(41 \%)$ \\
\hline Nephropathy & $9(16 \%)$ \\
\hline Neuropathy & $15(27 \%)$ \\
\hline \multicolumn{2}{|c|}{ Concomitant medication and therapies } \\
\hline \multicolumn{2}{|l|}{ Diabetes related } \\
\hline Metformin & $48(86 \%)$ \\
\hline Sulphonylurea & $15(27 \%)$ \\
\hline Gliptin & $6(11 \%)$ \\
\hline Glitazone & $5(9 \%)$ \\
\hline GLP-1 analogues & $5(9 \%)$ \\
\hline SGLT2-inhibitors & $0(0 \%)$ \\
\hline Insulin & $27(48 \%)$ \\
\hline \multicolumn{2}{|l|}{ Antihypertensives } \\
\hline ACE inhibitor/ARB & $48(86 \%)$ \\
\hline Calcium channel blocker & $13(23 \%)$ \\
\hline Diuretic & $16(29 \%)$ \\
\hline Beta-blockers & $26(47 \%)$ \\
\hline Alpha-blockers & $5(9 \%)$ \\
\hline \multicolumn{2}{|l|}{ Lipid-lowering } \\
\hline Statin & $52(93 \%)$ \\
\hline Fibrate & $1(2 \%)$ \\
\hline Ezetimibe & $7(13 \%)$ \\
\hline
\end{tabular}

$A R B$ angiotensin receptor blocker, $F T_{4}$ free thyroxine, GLP-1 glucagon like peptide 1, SGLT2 sodium-glucose transporter 2, TSH thyroid stimulating hormone 


\section{Platelet aggregation responses}

Maximum aggregation (MA) responses are summarised in Tables 2, 3 and Fig. 2a. Between the 3 treatments, there were significant differences in MA responses to all the agonists and concentrations tested (all $\mathrm{p}<0.001$ ). ADP-induced platelet aggregation, at all 5 concentrations tested, was significantly greater when receiving aspirin compared to clopidogrel (all $\mathrm{p}<0.001$ ) and clopidogrel compared to prasugrel (all $\mathrm{p}<0.001$ ). In contrast, platelet aggregation responses to $1 \mathrm{mmol} / \mathrm{L}$ AA were significantly lower when receiving aspirin $(6.6 \pm 19.0 \%)$ compared to clopidogrel $(63.4 \pm 34.6 \%, \mathrm{p}<0.001)$ and prasugrel $(52.6 \% \pm 31.1 \%, \mathrm{p}<0.001)$. The difference between clopidogrel and prasugrel was also significant $(p=0.027)$. The response to collagen $2 \mu \mathrm{g} / \mathrm{mL}$ was significantly reduced when receiving aspirin $(62.1 \pm 19.4 \%)$ compared to clopidogrel $(72.3 \pm 18.2 \%, \mathrm{p}=0.001)$, while prasugrel-treated individuals had a similar response to those on aspirin $(60.2 \pm 18.5 \%, \mathrm{p}>0.99)$. The response to collagen $16 \mu \mathrm{g} /$ $\mathrm{mL}$ was similar when receiving aspirin $(84.4 \pm 7.0 \%) \mathrm{com}-$ pared to clopidogrel $(83.8 \pm 8.1 \%, \mathrm{p}>0.99)$ but was lower when receiving prasugrel $(78.6 \pm 9.4 \%, \mathrm{p}<0.001)$. Compared to prasugrel, responses to both 2 and $16 \mu \mathrm{g} / \mathrm{mL}$ collagen were more pronounced when receiving clopidogrel $(\mathrm{p}<0.001$ and 0.003 respectively). FA responses followed a similar pattern (data not shown).

Comparing platelet aggregation responses when receiving standard-of-care aspirin $75 \mathrm{mg}$ OD to those at the end of the study aspirin period, there were no differences (Additional file 1: Table S1).

\section{High residual platelet reactivity}

High on-treatment residual platelet reactivity (HRPR), which has been associated with increased ischaemic

Table 2 Results of study endpoints relating to platelet function, P-selectin expression, fibrin clot dynamics, coagulationassociated proteins and inflammatory markers

\begin{tabular}{|c|c|c|c|c|c|}
\hline & & $\begin{array}{l}\text { Aspirin }(n=56) \\
\text { Mean } \pm \text { SD }\end{array}$ & $\begin{array}{l}\text { Clopidogrel }(n=56) \\
\text { Mean } \pm \text { SD }\end{array}$ & $\begin{array}{l}\text { Prasugrel }(n=56) \\
\text { Mean } \pm S D\end{array}$ & $\mathrm{p}$ \\
\hline \multicolumn{6}{|c|}{ Maximum platelet aggregation response (\%) } \\
\hline Agonist & [Agonist] & & & & \\
\hline \multirow[t]{5}{*}{ ADP } & $1 \mu \mathrm{mol} / \mathrm{L}$ & $42.7 \pm 15.6$ & $22.3 \pm 12.9$ & $11.5 \pm 8.1$ & $<0.001$ \\
\hline & $2 \mu \mathrm{mol} / \mathrm{L}$ & $61.0 \pm 12.4$ & $37.9 \pm 17.0$ & $21.0 \pm 11.4$ & $<0.001$ \\
\hline & $5 \mu \mathrm{mol} / \mathrm{L}$ & $71.0 \pm 8.9$ & $49.2 \pm 17.0$ & $27.9 \pm 12.8$ & $<0.001$ \\
\hline & $10 \mu \mathrm{mol} / \mathrm{L}$ & $80.5 \pm 9.7$ & $58.2 \pm 18.1$ & $33.6 \pm 14.3$ & $<0.001$ \\
\hline & $20 \mu \mathrm{mol} / \mathrm{L}$ & $77.6 \pm 8.4$ & $57.7 \pm 17.6$ & $34.1 \pm 14.1$ & $<0.001$ \\
\hline AA & $1 \mathrm{mmol} / \mathrm{L}$ & $6.6 \pm 19.0$ & $63.4 \pm 34.6$ & $52.6 \pm 31.1$ & $<0.001$ \\
\hline \multirow[t]{2}{*}{ Collagen } & $2 \mu \mathrm{g} / \mathrm{mL}$ & $62.1 \pm 19.4$ & $72.3 \pm 18.2$ & $60.2 \pm 18.5$ & $<0.001$ \\
\hline & $16 \mu \mathrm{g} / \mathrm{mL}$ & $84.4 \pm 7.0$ & $83.8 \pm 8.1$ & $78.6 \pm 9.4$ & $<0.001$ \\
\hline \multicolumn{6}{|c|}{ Platelet P-selectin expression (\%) } \\
\hline \multicolumn{6}{|l|}{$[A D P](\mu \mathrm{mol} / \mathrm{L})$} \\
\hline 0.3 & & $18.4 \pm 13.0$ & $12.5 \pm 10.4$ & $5.9 \pm 5.0$ & $<0.001$ \\
\hline 1 & & $31.2 \pm 19.0$ & $18.2 \pm 14.5$ & $9.0 \pm 6.8$ & $<0.001$ \\
\hline 3 & & $37.8 \pm 23.1$ & $23.5 \pm 17.3$ & $10.4 \pm 7.7$ & $<0.001$ \\
\hline 10 & & $41.5 \pm 23.7$ & $24.2 \pm 17.6$ & $11.5 \pm 8.1$ & $<0.001$ \\
\hline 30 & & $45.1 \pm 21.4$ & $27.1 \pm 19.0$ & $14.1 \pm 14.9$ & $<0.001$ \\
\hline \multicolumn{6}{|l|}{ Fibrin clot dynamics } \\
\hline Lag time & $(\mathrm{s})$ & $683.3 \pm 170.4$ & $706.9 \pm 196.2$ & $665.0 \pm 151.8$ & 0.007 \\
\hline Maximum absorbance & $(A U)$ & $0.2 \pm 0.08$ & $0.2 \pm 0.09$ & $0.2 \pm 0.08$ & 0.65 \\
\hline Lysis time & $(\mathrm{s})$ & $519.6 \pm 112.3$ & $522.3 \pm 132.8$ & $522.4 \pm 101.2$ & 0.95 \\
\hline \multicolumn{6}{|c|}{ Coagulation-associated proteins } \\
\hline Fibrinogen & $(g / L)$ & $2.8 \pm 0.5$ & $2.7 \pm 0.5$ & $2.7 \pm 0.5$ & 0.69 \\
\hline PAl-1 & $(p g / m L)$ & $2334.6 \pm 1675.3$ & $2132.5 \pm 1626.4$ & $2089.3 \pm 1667.1$ & 0.27 \\
\hline \multicolumn{6}{|l|}{ Inflammatory markers } \\
\hline WCC & $\left(\times 10^{6} / L\right)$ & $7.2 \pm 1.8$ & $6.7 \pm 1.9$ & $7.0 \pm 2.0$ & 0.067 \\
\hline CRP & $(\mathrm{mg} / \mathrm{L})$ & $2.3 \pm 3.4$ & $2.4 \pm 4.6$ & $1.8 \pm 2.2$ & 0.5 \\
\hline $\mathrm{C} 3$ & $(\mathrm{~g} / \mathrm{L})$ & $0.7 \pm 0.1$ & $0.7 \pm 0.1$ & $0.7 \pm 0.1$ & 0.27 \\
\hline
\end{tabular}

$\mathrm{p}$ values represent a repeated-measures ANOVA conducted between the three treatments

$A A$ arachidonic acid, C3 complement fragment 3, PAI-1 plasminogen activator inhibitor 1, WCC white cell count 
Table 3 Pairwise comparisons (with Bonferroni correction) for those endpoints with significant differences between the three treatments on ANOVA

\begin{tabular}{|c|c|c|c|c|c|c|c|}
\hline & & \multicolumn{2}{|l|}{ Aspirin vs. clopidogrel } & \multicolumn{2}{|l|}{ Aspirin vs. prasugrel } & \multicolumn{2}{|l|}{ Clopidogrel vs. prasugrel } \\
\hline & & Mean difference $(95 \% \mathrm{Cl})$ & p & Mean difference $(95 \% \mathrm{Cl})$ & $\mathbf{p}$ & Mean difference $(95 \% \mathrm{Cl})$ & $\mathbf{p}$ \\
\hline \multicolumn{8}{|c|}{ Maximum platelet aggregation response (\%) } \\
\hline Agonist & Concentratio & & & & & & \\
\hline \multirow[t]{5}{*}{ ADP } & $1 \mu \mathrm{mol} / \mathrm{L}$ & 20.46 (16.33 to 24.58$)$ & $<0.001$ & 31.24 (26.79 to 35.68$)$ & $<0.001$ & 10.78 (7.34 to 14.22$)$ & $<0.001$ \\
\hline & $2 \mu \mathrm{mol} / \mathrm{L}$ & 23.04 (17.17 to 29.91) & $<0.001$ & 39.96 (35.75 to 44.18) & $<0.001$ & 16.93 (12.48 to 21.00$)$ & $<0.001$ \\
\hline & $5 \mu \mathrm{mol} / \mathrm{L}$ & 21.80 (16.01 to 27.59$)$ & $<0.001$ & 43.07 (38.54 to 47.60$)$ & $<0.001$ & 21.27 (16.75 to 25.80$)$ & $<0.001$ \\
\hline & $10 \mu \mathrm{mol} / \mathrm{L}$ & 22.29 (15.97 to 28.61$)$ & $<0.001$ & 46.86 (41.39 to 52.32$)$ & $<0.001$ & 24.56 (28.98 to 20.14) & $<0.001$ \\
\hline & $20 \mu \mathrm{mol} / \mathrm{L}$ & 19.94 (13.71 to 26.14) & $<0.001$ & 43.53 (38.57 to 48.49$)$ & $<0.001$ & 23.60 (19.34 to 27.83$)$ & $<0.001$ \\
\hline AA & $1 \mathrm{mmol} / \mathrm{L}$ & $-56.86(-68.89$ to -44.82$)$ & $<0.001$ & $-45.98(-56.77$ to -35.20$)$ & $<0.001$ & 10.87 (0.95 to 20.79) & 0.027 \\
\hline \multirow[t]{2}{*}{ Collagen } & $2 \mu \mathrm{g} / \mathrm{mL}$ & $-10.24(-16.52$ to -3.95$)$ & 0.001 & $1.86(-3.22$ to 6.92$)$ & $>0.99$ & 12.09 (7.52 to 16.66$)$ & $<0.001$ \\
\hline & $16 \mu \mathrm{g} / \mathrm{mL}$ & $0.62(-2.28$ to 3.52$)$ & $>0.99$ & 5.73 (2.71 to 8.74$)$ & $<0.001$ & 5.11 (1.48 to 8.74$)$ & 0.003 \\
\hline \multicolumn{8}{|c|}{ Platelet P-selectin expression (\%) } \\
\hline \multicolumn{8}{|l|}{$[A D P] \mu m o / / L$} \\
\hline 0.3 & & 5.87 (2.05 to 9.69$)$ & 0.001 & 12.52 (8.32 to 16.72$)$ & $<0.001$ & 6.65 (3.65 to 9.65) & $<0.001$ \\
\hline 1 & & 13.00 (6.56 to 19.43$)$ & $<0.001$ & 22.23 (16.00 to 28.45$)$ & $<0.001$ & $9.23(5.30$ to 13.16$)$ & $<0.001$ \\
\hline 3 & & 14.27 (6.95 to 21.58) & $<0.001$ & 27.37 (19.63 to 35.11$)$ & $<0.001$ & 13.10 (8.19 to 18.01$)$ & $<0.001$ \\
\hline 10 & & 17.28 (9.90 to 24.66$)$ & $<0.001$ & 29.95 (22.17 to 37.74$)$ & $<0.001$ & 12.67 (7.55 to 17.80$)$ & $<0.001$ \\
\hline 30 & & 18.02 (9.82 to 26.22) & $<0.001$ & 30.97 (23.13 to 38.81) & $<0.001$ & 12.95 (7.22 to 18.68$)$ & $<0.001$ \\
\hline \multicolumn{8}{|c|}{ Fibrin clot dynamics } \\
\hline Lag time ( & & $-23.61(-56.2$ to 8.98$)$ & 0.237 & $18.28(-8.57$ to 45.12$)$ & 0.295 & 41.8 (7.41 to 76.37$)$ & 0.012 \\
\hline
\end{tabular}

$A A$ arachidonic acid
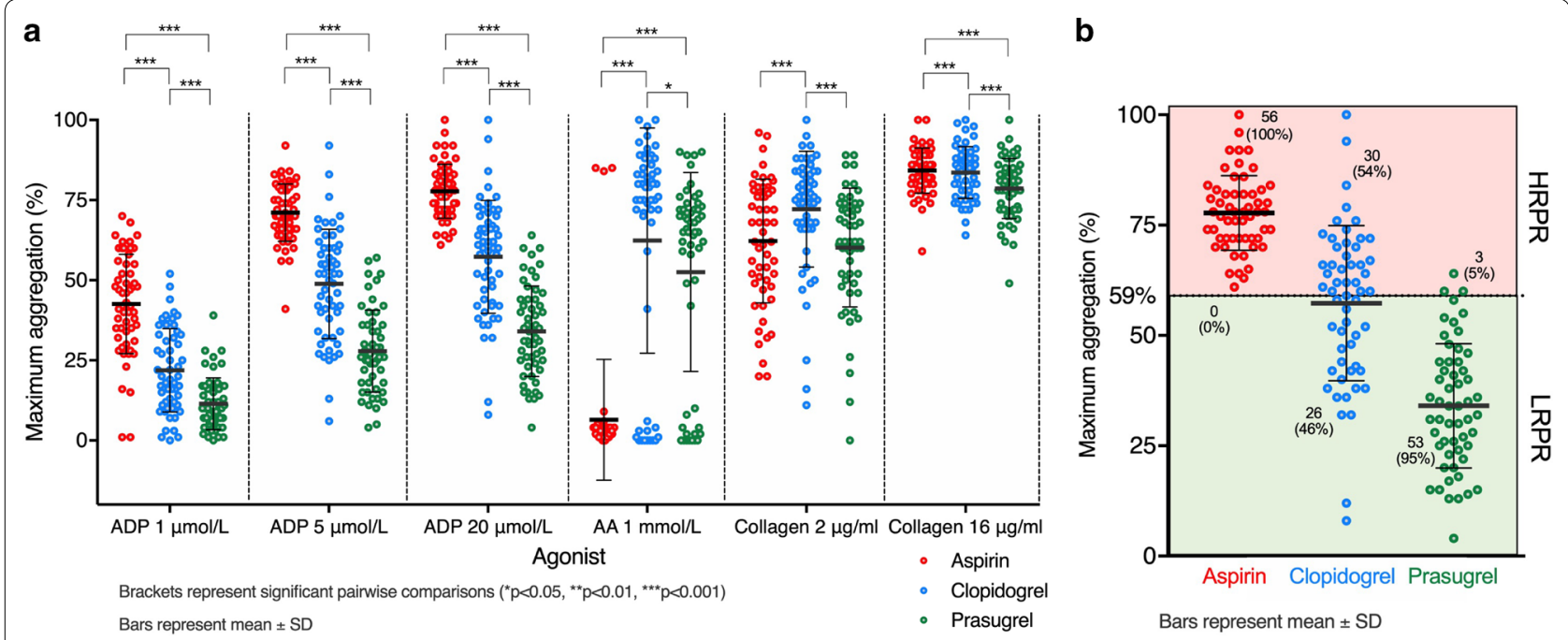

Fig. 2 a Maximum platelet aggregation responses assessed by light transmittance aggregometry. $A A$ arachidonic acid; $\mathbf{b}$ proportions of participants during each treatment period with high (HRPR) and low residual platelet reactivity (LRPR), defined as a maximum aggregation response to $20 \mu \mathrm{mol} / \mathrm{L}$ ADP of $>59 \%$ and $\leq 59 \%$ respectively

risk, can be defined based on a maximum aggregatory response to $20 \mu \mathrm{mol} / \mathrm{L}$ ADP of $>59 \%$, when assessed by LTA [36-38]. In this study, whilst receiving aspirin, all participants had HRPR. The proportion was reduced compared to aspirin when receiving either clopidogrel (relative risk [RR] 0.54, 95\% CI [0.41-0.66], p < 0.0001) or prasugrel (RR $0.05[0.02-0.15], \mathrm{p}<0.0001$ ), and when receiving prasugrel compared to clopidogrel (RR 0.1 
[0.03-0.28], $\mathrm{p}<0.0001$ ) (Additional file 1: Table S2, Fig. 2b).

\section{Platelet P-selectin expression}

Measurement of ADP-stimulated platelet P-selectin expression revealed significant differences between the 3 treatments at all concentrations of ADP used (e.g. 30 $\mu \mathrm{mol} / \mathrm{L}$ : aspirin $45.1 \pm 21.4 \%$ vs. clopidogrel $27.1 \pm 19.0 \%$ vs. prasugrel $14.1 \pm 14.9 \%, \mathrm{p}<0.001$ ) (Table 2, Fig. 3). On pairwise comparison, expression was significantly greater when treated with clopidogrel vs. aspirin, prasugrel vs. aspirin and prasugrel vs. clopidogrel (all $\mathrm{p} \leq 0.001$ ) (Table 3, Fig. 3).

\section{Fibrin clot properties}

Lag time was significantly different when comparing aspirin, clopidogrel and prasugrel treatment $(683.3 \pm 170.4 \mathrm{~s}$ vs. $706.9 \pm 196.2 \mathrm{~s}$ vs. $665.0 \pm 151.8 \mathrm{~s}$, respectively, $\mathrm{p}=0.007$ [ANOVA]) (Table 2, Additional file 1: Figure $\mathrm{S} 1)$. There was no difference between aspirin and clopidogrel $(\mathrm{p}=0.24)$, nor aspirin and prasugrel $(\mathrm{p}=0.30)$, but lag time was significantly longer when receiving clopidogrel vs. prasugrel $(\mathrm{p}=0.012)$ (Table 3, Additional file 1: Figure S2). There were no significant differences between the treatments in final clot turbidity $(0.2 \pm 0.08$ (arbitrary units) vs. $0.2 \pm 0.09$ vs. $0.2 \pm 0.08, \mathrm{p}=0.65$ ) or lysis time $(519.6 \pm 112.3$ s vs. $522.3 \pm 132.8$ s vs. $522.4 \pm 101.2$, $\mathrm{p}=0.95$ ) (Table 2, Additional file 1: Figure S3). Lysis time, but not other parameters, significantly correlated with $\mathrm{HbA1}_{\mathrm{c}}(\mathrm{R}=0.18, \mathrm{p}=0.027)$ (Additional file 1: Table S3).

\section{Coagulation factors and inflammatory markers}

No significant differences in fibrinogen, circulating leukocyte count, CRP or complement C3 were observed between the treatments (all $\mathrm{p}>0.05$, Table 2 ).

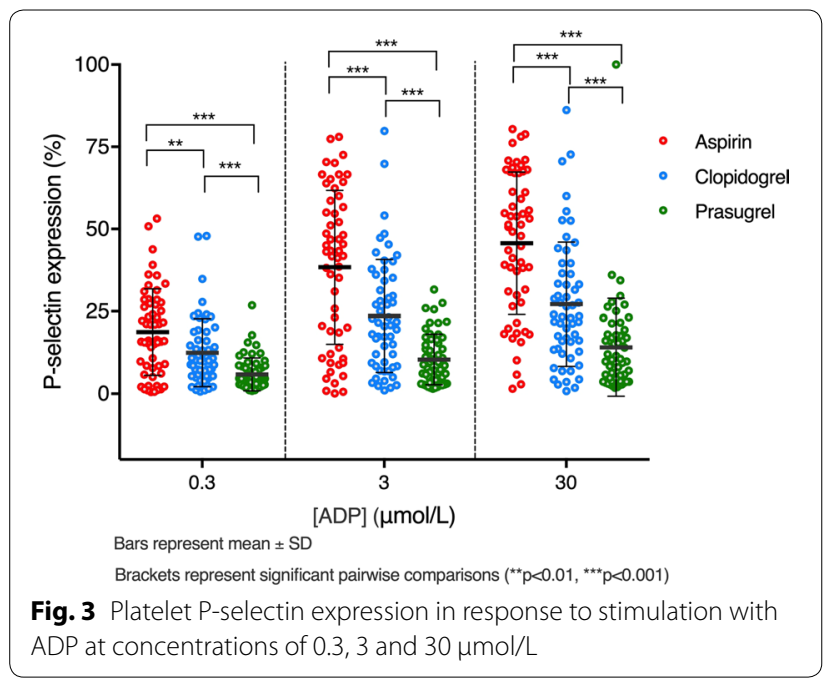

\section{MiRNA quantification}

Significant differences were seen between the treatments in circulating levels of miR-21, miR-24, miR-191, miR197 and miR-223 (Table 4, Fig. 4). Post-hoc pairwise comparisons revealed significantly lower miRNA expression, when receiving prasugrel compared to aspirin, of miR-24 $(\mathrm{p}=0.004), \mathrm{miR}-191(\mathrm{p}=0.019), \mathrm{miR}-197(\mathrm{p}=0.009)$ and miR-223 $(\mathrm{p}=0.014)$, but not miR-21 $(\mathrm{p}=0.10$ (Table 5 , Fig. 5). There were no significant differences in miRNA levels between aspirin and clopidogrel nor between clopidogrel and prasugrel.

\section{Correlation between miRNA detection and other markers}

Platelet ADP-stimulated P-selectin expression correlated with circulating levels of miR-21 $(R=0.23$, $\mathrm{p}=0.003), \quad \operatorname{miR}-24 \quad(\mathrm{R}=0.22, \quad \mathrm{p}=0.004), \quad \operatorname{miR}-191$ $(\mathrm{R}=0.2, \mathrm{p}=0.008)$ and $\mathrm{miR}-223(\mathrm{R}=0.25, \mathrm{p}=0.002)$, but not miR-197 ( $\mathrm{R}=0.12, \mathrm{p}=0.13)$ (Fig. 5, Additional file 1: Table S3). Conversely, there was a negative correlation between AA-induced platelet aggregation and levels of miR-24 $(\mathrm{R}=-0.21, \mathrm{p}=0.004)$, miR-191 $(\mathrm{R}=-0.20, \mathrm{p}=0.01), \mathrm{miR}-197(\mathrm{R}=-0.23, \mathrm{p}=0.002)$ and miR-223 $(\mathrm{R}=-0.24, \mathrm{p}=0.002)$ but not miR-21 $(\mathrm{R}=-0.08, \mathrm{p}=0.30)$ (Additional file 1: Table S3). No significant correlations were observed between ADP- or collagen-induced platelet aggregation and circulating miRNA levels (Additional file 1: Table S3).

Of the fibrin clot parameters studied, there was a significant positive correlation between final clot turbidity

Table 4 Levels of circulating miRNAs in patients with diabetes receiving aspirin, clopidogrel or prasugrel. Values shown are mean \pm SD. $P$ values were generated by one-way repeated measures ANOVA with GreenhouseGeisser correction

\begin{tabular}{llllll}
\hline $\mathbf{m i R}$ & \multicolumn{2}{l}{$\mathbf{2}^{-(\mathbf{\Delta} \mathbf{\Delta C q})}(\mathbf{m e a n} \pm \mathbf{S D})$} & $\mathbf{p}$ & $\begin{array}{l}\text { Partial } \mathbf{\eta}^{\mathbf{2}} \\
\text { (effect size) }\end{array}$ \\
\cline { 2 - 4 } & Aspirin & Clopidogrel & Prasugrel & & \\
\hline 21 & $1.21 \pm 0.67$ & $1.04 \pm 0.51$ & $1.03 \pm 0.47$ & 0.028 & 0.065 \\
24 & $1.05 \pm 0.64$ & $0.88 \pm 0.70$ & $0.75 \pm 0.40$ & 0.009 & 0.084 \\
$27 \mathrm{~b}$ & $1.09 \pm 0.79$ & $0.96 \pm 1.22$ & $0.75 \pm 0.38$ & 0.115 & 0.042 \\
28 & $0.83 \pm 0.54$ & $0.65 \pm 0.53$ & $0.63 \pm 0.60$ & 0.083 & 0.046 \\
93 & $1.06 \pm 0.60$ & $0.95 \pm 0.74$ & $1.01 \pm 0.78$ & 0.662 & 0.007 \\
122 & $0.93 \pm 0.82$ & $0.98 \pm 0.98$ & $1.07 \pm 1.00$ & 0.278 & 0.023 \\
126 & $0.93 \pm 0.42$ & $0.83 \pm 0.47$ & $0.80 \pm 0.34$ & 0.110 & 0.057 \\
150 & $0.83 \pm 0.34$ & $0.81 \pm 0.43$ & $0.80 \pm 0.36$ & 0.777 & 0.004 \\
191 & $0.87 \pm 0.65$ & $0.66 \pm 0.62$ & $0.59 \pm 0.48$ & 0.017 & 0.073 \\
197 & $1.13 \pm 0.68$ & $0.97 \pm 0.82$ & $0.82 \pm 0.45$ & 0.019 & 0.072 \\
223 & $0.93 \pm 0.55$ & $0.78 \pm 0.50$ & $0.70 \pm 0.41$ & 0.014 & 0.076 \\
320 & $0.93 \pm 0.46$ & $0.82 \pm 0.46$ & $0.79 \pm 0.40$ & 0.087 & 0.045 \\
$451 \mathrm{a}$ & $1.04 \pm 0.65$ & $0.95 \pm 0.43$ & $1.20 \pm 0.99$ & 0.165 & 0.034 \\
486 & $0.96 \pm 0.70$ & $0.85 \pm 0.45$ & $1.11 \pm 1.17$ & 0.215 & 0.028 \\
\hline & & & & & \\
\hline
\end{tabular}




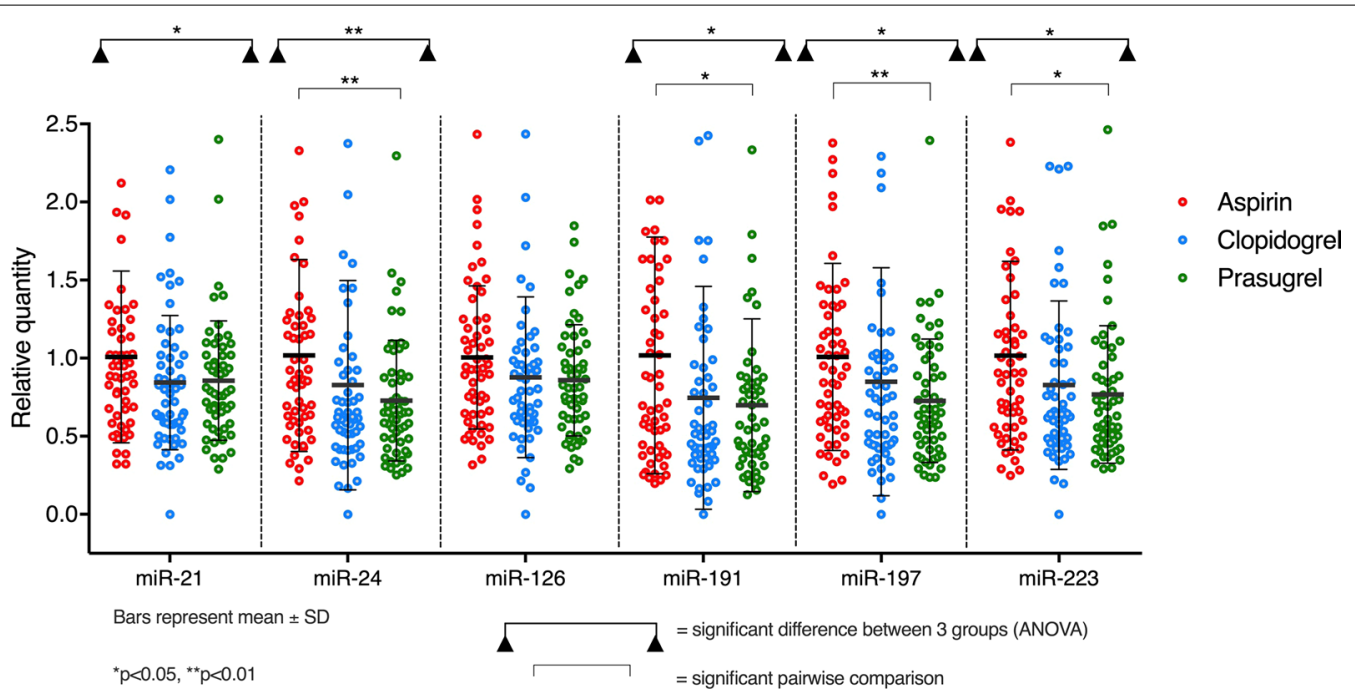

Fig. 4 Quantification of plasma levels of miR-21, miR-24, miR-126, miR-191, miR-197 and miR-223 using the $2^{-\Delta \Delta c a}$ method, expressed relative to the mean value when receiving aspirin

Table 5 Pairwise comparisons (with Bonferroni correction) for those miRNAs with significant differences between the three treatments on ANOVA

\begin{tabular}{|c|c|c|c|c|c|c|}
\hline \multirow[t]{2}{*}{ miR- } & \multicolumn{2}{|l|}{ Aspirin vs. clopidogrel } & \multicolumn{2}{|l|}{ Aspirin vs. prasugrel } & \multicolumn{2}{|l|}{ Clopidogrel vs. prasugrel } \\
\hline & Mean difference $(95 \% \mathrm{Cl})$ & $p$ & Mean difference $(95 \% \mathrm{Cl})$ & $\mathbf{p}$ & Mean difference $(95 \% \mathrm{Cl})$ & $\mathrm{p}$ \\
\hline 21 & $0.17(-0.004$ to 0.345$)$ & 0.058 & $0.177(-0.023$ to 0.378$)$ & 0.100 & 0.007 ( -0.158 to 0.172$)$ & $>0.99$ \\
\hline 24 & $0.165(-0.069$ to 0.398$)$ & 0.262 & 0.295 (0.081 to 0.510$)$ & 0.004 & $0.131(-0.116$ to 0.377$)$ & 0.586 \\
\hline 191 & $0.208(-0.031$ to 0.446$)$ & 0.108 & 0.272 (0.036 to 0.508$)$ & 0.019 & $0.064(-0.185$ to 0.313$)$ & $>0.99$ \\
\hline 197 & $0.153(-0.138$ to 0.444$)$ & 0.596 & 0.311 (0.065 to 0.558) & 0.009 & $0.158(-0.102$ to 0.418$)$ & 0.416 \\
\hline 223 & $0.146(-0.052$ to 0.343$)$ & 0.220 & $0.226(0.036$ to 0.416$)$ & 0.014 & $0.08(-0.103$ to 0.263$)$ & 0.852 \\
\hline
\end{tabular}

$\mathrm{Cl}$ confidence interval

and $\mathrm{miR}-21(\mathrm{R}=0.22, \mathrm{p}=0.006$, Additional file 1: Figure S3) but no other parameters, nor with other miRNAs (Additional file 1: Table 3). Subgroup analysis of levels miR-21, miR-24, miR-191, miR-197 and miR223 by HRPR status revealed no significant differences between the groups (Additional file 1: Table S4).

\section{miR-126}

Although levels of miR-126 have been linked to platelet and endothelial function in the general population [25], T2DM may be associated with lower detectable quantities [39]. P-selectin expression in response to ADP stimulation showed positive correlation with miR-126 $(\mathrm{R}=0.27, \mathrm{p}=0.0004)$ (Additional file 1: Table S3, Additional file 1: Figure S4). On the other hand, we failed to show significant differences in quantification of miR-126 between the treatments and there was no evidence of a significant correlation between aggregation responses and miR-126 (Additional file 1: Table S2).
Effect of presence or absence of cardiovascular disease Subgroup analysis by presence $(\mathrm{n}=32)$ or absence $(n=24)$ of a history of macrovascular atheromatous disease (history of coronary artery disease, cerebrovascular ischaemia or peripheral arterial disease) at enrolment revealed no significant differences between the subgroups in markers of platelet aggregation during each of the three treatment periods (Additional file 1: Table S5). Of the five miRNAs identified as having different expression profiles across the treatments, levels of miR-197 were significantly lower in those with cardiovascular disease compared to those without when receiving aspirin $(0.97 \pm 0.63$ vs. $1.35 \pm 0.69, \mathrm{p}=0.04)$ and prasugrel $(0.68 \pm 0.33$ vs. $0.99 \pm 0.46, p=0.008)$, but not clopidogrel $(0.85 \pm 0.78$ vs. $1.15 \pm 0.89, \mathrm{p}=0.2)$ (Additional file 1 : Table S5, Fig. 6). There were no significant differences between quantification of other miRNAs and cardiovascular disease state (Additional file 1: Table S5), including miR-126 (Additional file 1: Figure S6). 

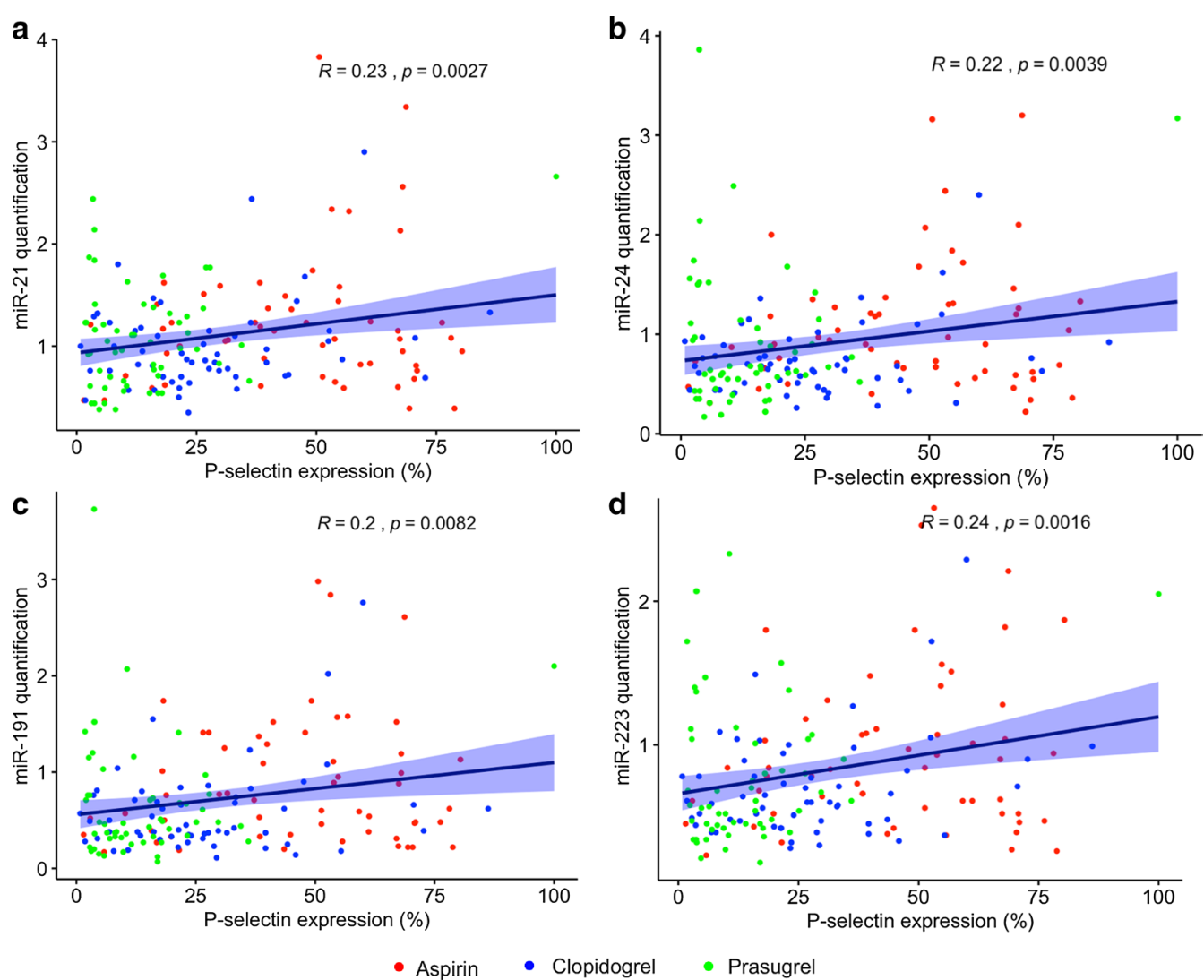

Fig. 5 Correlation between platelet P-selectin expression, after stimulation with $30 \mu \mathrm{mol} / \mathrm{L}$ ADP, and relative quantity of a miR-21, b miR24, c miR-191 and d miR-223. Dark blue lines indicate those of best linear fit, light blue shading indicates $95 \%$ confidence interval. R and $p$ values were produced by Pearson correlation analysis

\section{Discussion}

Patients with T2DM present particular challenges with regards to atherothrombotic protection. There is no current clear strategy of antiplatelet therapy for primary prevention in those with T2DM. For example, whilst some evidence suggests that aspirin therapy targeted by assessment of cardiovascular and bleeding risk may be of benefit [40], guidelines remain conflicted on the extent to which, if at all, aspirin therapy should be recommended in this situation $[41,42]$. $\mathrm{P}_{2} \mathrm{Y}_{12}$ inhibitors are alternative antiplatelet agents to aspirin with the potential benefits of prolonged presence in the plasma that might overcome reduced aspirin effect due to high platelet turnover and avoiding gastric erosion [43]. Three orally-active drugs, clopidogrel, prasugrel and ticagrelor are commonly-available [6]. Monotherapy with clopidogrel offers only modest clinical benefits over aspirin in the setting of secondary prevention, but these may be amplified in those with T2DM [11]. Furthermore, the newer $\mathrm{P}_{2} \mathrm{Y}_{12}$ inhibitors ticagrelor and prasugrel are more potent and consistent in effect than clopidogrel [44]. Certainly, when given in combination

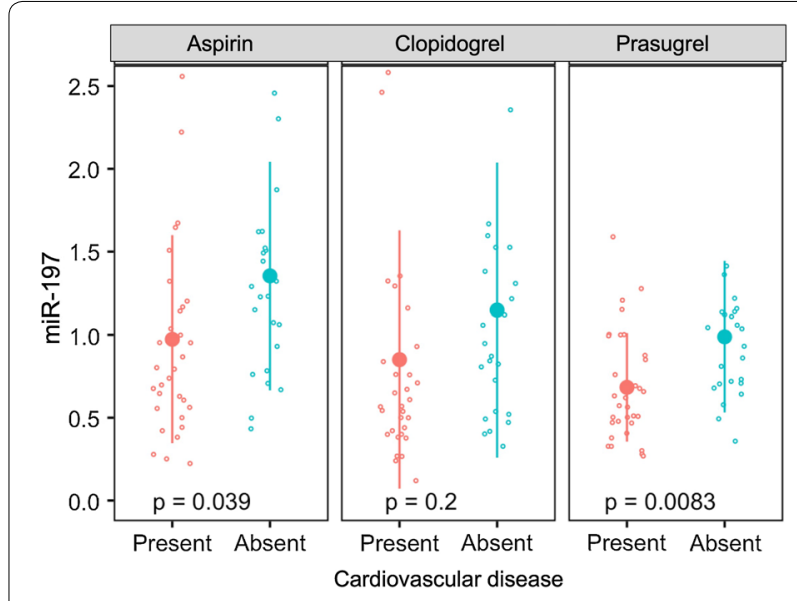

Fig. 6 Relative quantification of circulating miR-197 in participants with and without a history of cardiovascular disease during the three treatment periods. Large dots and lines represent mean \pm SD. $p$ values were generated by t-tests

with aspirin, ticagrelor and prasugrel offer net clinical benefit after ACS [6]. Additionally, there is evidence that potent $\mathrm{P} 2 \mathrm{Y}_{12}$ inhibition may offer benefits in 
patients with T2DM and complications such as lower extremity arterial disease, in which ticagrelor improves microvascular flow, for example [45]. Although there has been concern over greater bleeding risk increasing the potency of $\mathrm{P}_{2} \mathrm{Y}_{12}$ inhibition, recent evidence suggests, for example, ticagrelor may have similar safety to clopidogrel in patients at high risk of bleeding, such as those who are elderly with ST-elevation myocardial infarction [46], and low-dose prasugrel appears to be of comparable safety to clopidogrel when used in triple therapy (aspirin, $\mathrm{P}_{2} \mathrm{Y}_{12}$ inhibitor and anticoagulant) for patients with atrial fibrillation undergoing percutaneous coronary intervention [47].

In this study, we have comprehensively characterised the effects of aspirin, clopidogrel and prasugrel when given as SAPT to patients with T2DM. Prasugrel provided the strongest effect on ADP-induced platelet aggregation, as did aspirin on AA-induced aggregation. Prasugrel also provided greater and more consistent inhibition of ADP-induced platelet aggregation when compared to clopidogrel, in agreement with previous studies on populations with and without diabetes receiving dual antiplatelet therapy $[44,48]$ and of studies of prasugrel vs. clopidogrel loading doses when given as SAPT [49]. In concert with this was the fact that there was a large reduction in the proportion of patients with HRPR when receiving clopidogrel vs. aspirin, and again when receiving prasugrel vs. either comparator. P-selectin expression after stimulation with ADP followed a similar pattern to the aggregation responses. Collagen-induced platelet aggregation, perhaps representing the best global assessment of effects on platelet macroaggregation, was more strongly inhibited by prasugrel than aspirin and clopidogrel, suggesting that prasugrel acts as the most potent antiplatelet drug of the three when used as monotherapy in patients with T2DM.

For the first time, we studied a panel of miRNAs in patients with diabetes receiving three different antiplatelet agents, showing that potent $\mathrm{P}_{2} \mathrm{Y}_{12}$ inhibition with prasugrel reduced detectable levels of miR-24, miR-191, miR-197 and miR-223 compared to aspirin. Although there were no significant differences between aspirin and clopidogrel nor clopidogrel and prasugrel, there did appear to be similar trends; thienopyridines reduced miRNA levels, an effect that was most pronounced with prasugrel. The known actions and associations of miRNAs are broad-ranging, but circulating levels of miR-21, miR-24, miR-197 and miR-223 are most strongly associated with platelets and platelet microparticles, along with miR-126 [26]. These, as well as miR-191, have been shown to be reduced by antiplatelet therapy in healthy volunteers, and some also in patients with cardiovascular disease [24, 25]. Our study, in individuals with T2DM, supports the association between plasma levels of these miRNAs and platelet function.

Whilst there were positive correlations between miRNA levels and ADP-induced platelet P-selectin expression, there was no evidence of a significant correlation with ADP or collagen-induced aggregation, and we observed negative correlations with AA-induced aggregation in some cases. P-selectin expression, which is known to be reduced by $\mathrm{P}_{2} \mathrm{Y}_{12}$ inhibitors but not aspirin [28], occurs when alpha granules fuse with the cell membrane upon platelet activation. These data suggest that plasma levels of miRNAs reflect primarily the tendency for platelet degranulation and indeed it has previously been shown that miRNAs may be involved in regulating degranulation [50]. This has not been reported before in individuals with diabetes receiving antiplatelet therapy and future research is required to understand the specific role of each of the miRNAs, which may help with risk stratification and/or uncover alternative therapeutic targets to control platelet activation in this population.

We saw lower plasma quantities of miR-197 in T2DM patients with known cardiovascular disease compared to those without, which is consistent with the previous finding in a general population cohort suggesting that lower miR-197 might be associated with increased risk of myocardial infarction $[26,51]$. In contrast to previous findings in the general population, we did not see evidence of an association between elevated levels of miR-126 and presence of cardiovascular disease. Notably, however, studies have suggested that T2DM itself is associated with lower detectable quantities of miR-126 and therefore its prognostic significance as a vascular marker is potentially lost in the presence of diabetes and this may further explain the failure to demonstrate a treatment effect [39].

\section{Conclusion}

In summary, our data suggest that prasugrel monotherapy is superior to either aspirin or clopidogrel in inhibiting platelet function in diabetes. From a translational perspective these findings could have the potential to be implemented in personalised treatment options for patients with T2DM and cardiovascular disease. Moreover, our miRNA results indicate that assessing response to antiplatelet therapy does not necessarily require fresh blood samples and tests can be conducted on miRNA measurements as biomarkers from stored acellular plasma samples. miRNA measurements may provide a platform to identify patients at greater risk of ischaemic heart disease relating to their platelet function. Based on these data and acknowledging that platelet activation is the central process in the development of 
atherothrombosis, a trial assessing the effects on clinical outcomes of prasugrel monotherapy may be warranted for the primary or secondary prevention of ischaemic heart disease in patients with T2DM. Also, further analysing the role of miRNA in predicting vascular outcome in individuals with diabetes may offer a tool to measure the clinical efficacy of antiplatelet agents.

\section{Supplementary information}

Supplementary information accompanies this paper at https://doi. org/10.1186/s12933-019-0981-3.

Additional file 1. Additional figures and tables

\section{Abbreviations}

AA: arachidonic acid; AU: absorbance units; ACS: acute coronary syndrome; CAPRIE: clopidogrel vs aspirin in patients at risk of ischaemic events; Cq: quantification cycle; FA: final aggregation; HRPR: high on-treatment residual platelet reactivity; MA: maximum aggregation; miRNA: micro ribonucleic acid; OD: once-daily; P2Y $_{12}$ : P2Y purinoceptor type 12 (platelet adenosine diphosphate receptor); RT-qPCR: reverse transcription quantitative polymerase chain reaction; RNA: ribonucleic acid; SAPT: single antiplatelet therapy; T2DM: type 2 diabetes mellitus.

\section{Acknowledgements}

W.A.E. Parker is funded by a British Heart Foundation Clinical Training Research Fellowship (FS/18/49/33752). C. Schulte is the recipient of a research fellowship by the Deutsche Forschungsgemeinschaft (DFG) (SCHU 2983/1-1). T. Barwari is funded by a British Heart Foundation (BHF) Interdisciplinary Ph.D. studentship. M. Mayr is a BHF Chair Holder (CH/16/3/32406) with BHF programme Grant support (RG/16/14/32397).

\section{Authors' contributions}

The study was conceived and supervised by RAA, RFS and PJG. MM and TB designed and oversaw the microRNA analysis. SMP and RAA recruited patients and obtained study samples. Laboratory analyses were performed by FP, CS and TB. WAEP performed statistical analysis, collated results and produced the first draft of the manuscript. All authors read and approved the final manuscript.

\section{Funding}

This study was funded by Eli Lilly through an investigator-led grant. Eli Lilly was not involved in the design of the study; collection, analysis or interpretation of data; writing the report; or the decision to submit the report for publication.

\section{Availability of data and materials}

All data generated or analysed during this study are included in this published article and its Additional file 1.

\section{Ethics approval and consent to participate}

The study was approved by NHS Research Ethics Service (reference 09/ $\mathrm{H} 1307 / 110)$. Written consent was obtained from participants before any study activities took place.

\section{Consent for publication}

Not applicable.

\section{Competing interests}

M Mayr filed and licensed patent applications on miRNAs as biomarkers (EP15193448.6, EP2776580 B1, DE112013006129T5, GB2524692A, EP2576826 B, JP2013-513740). M. Mayr filed and licensed patent applications on miRNAs as biomarkers (EP15193448.6, EP2776580 B1, DE112013006129T5, GB2524692A, EP2576826 B, JP2013-513740).RF Storey reports institutional research grants/support from AstraZeneca and GlyCardial Diagnostics; consultancy fees from Amgen, AstraZeneca, Bayer, Bristol Myers Squibb/Pfizer, GlyCardial Diagnostics, Haemonetics, Idorsia, Novartis, Portola and Thromboserin; and honoraria from AstraZeneca, Bayer, Bristol Myers Squibb/Pfizer and Medscape. R. Ajjan. received honoraria and educational and research support from Abbott Diabetes Care, AstraZeneca, Novo Nordisk, Eli Lilly, Bayer, Sanofi, MSD, and Boehringer Ingelheim. The other authors report no relevant disclosures.

\section{Author details}

1 Department of Infection, Immunity \& Cardiovascular Disease, University of Sheffield, Sheffield, UK. ${ }^{2}$ King's British Heart Foundation Centre, King's College London, London, UK. ${ }^{3}$ Department of General and Interventional Cardiology, University Heart Centre Hamburg Eppendorf, Hamburg, Germany. ${ }^{4}$ Leeds Institute of Cardiovascular and Metabolic Medicine, University of Leeds, Leeds, UK.

Received: 10 November 2019 Accepted: 26 December 2019

Published online: 07 January 2020

References

1. Wendelboe AM, Raskob GE. Global burden of thrombosis: epidemiologic aspects. Circ Res. 2016;118(9):1340-7.

2. Grundy SM, Cleeman JI, Daniels SR, Donato KA, Eckel RH, Franklin BA, Gordon DJ, Krauss RM, Savage PJ, Smith SC Jr, et al. Diagnosis and management of the metabolic syndrome: an American Heart Association/ National Heart, Lung, and Blood Institute Scientific Statement. Circulation. 2005;112(17):2735-52.

3. Jackson SP. Arterial thrombosis-insidious, unpredictable and deadly. Nat Med. 2011:17(11):1423-36.

4. Kearney K, Tomlinson D, Smith K, Ajjan R. Hypofibrinolysis in diabetes: a therapeutic target for the reduction of cardiovascular risk. Cardiovasc Diabetol. 2017;16(1):34.

5. Rivas Rios JR, Franchi F, Rollini F, Angiolillo DJ. Diabetes and antiplatelet therapy: from bench to bedside. Cardiovasc Diagn Ther. 2018;8(5):594-609.

6. Parker WA, Storey RF. Long-term antiplatelet therapy following myocardial infarction: implications of PEGASUS-TIMI 54. Heart. 2016;102(10):783-9.

7. Yusuf S, Zhao F, Mehta SR, Chrolavicius S, Tognoni G, Fox KK. Effects of clopidogrel in addition to aspirin in patients with acute coronary syndromes without ST-segment elevation. N Engl J Med. 2001;345(7):494-502.

8. Wiviott SD, Braunwald E, McCabe CH, Montalescot G, Ruzyllo W, Gottlieb S, Neumann FJ, Ardissino D, De Servi S, Murphy SA, et al. Prasugrel versus clopidogrel in patients with acute coronary syndromes. N Engl J Med. 2007;357(20):2001-15.

9. Wiviott SD, Braunwald E, Angiolillo DJ, Meisel S, Dalby AJ, Verheugt FW, Goodman SG, Corbalan R, Purdy DA, Murphy SA, et al. Greater clinical benefit of more intensive oral antiplatelet therapy with prasugrel in patients with diabetes mellitus in the trial to assess improvement in therapeutic outcomes by optimizing platelet inhibition with prasugrelthrombolysis in myocardial infarction 38. Circulation. 2008;118:1626-36.

10. CAPRIE Steering Committee. A randomised, blinded, trial of clopidogrel versus aspirin in patients at risk of ischaemic events (CAPRIE). Lancet. 1996;348(9038):1329-39.

11. Bhatt DL, Marso SP, Hirsch AT, Ringleb PA, Hacke W, Topol EJ. Amplified benefit of clopidogrel versus aspirin in patients with diabetes mellitus. Am J Cardiol. 2002;90(6):625-8.

12. Belch J, MacCuish A, Campbell I, Cobbe S, Taylor R, Prescott R, Lee R, Bancroft J, MacEwan S, Shepherd J, et al. The prevention of progression of arterial disease and diabetes (POPADAD) trial: factorial randomised placebo controlled trial of aspirin and antioxidants in patients with diabetes and asymptomatic peripheral arterial disease. BMJ. 2008;337:1840.

13. Pignone M, Alberts MJ, Colwell JA, Cushman M, Inzucchi SE, Mukherjee D, Rosenson RS, Williams CD, Wilson PW, Kirkman MS. Aspirin for primary prevention of cardiovascular events in people with diabetes: a position statement of the american diabetes association, a scientific statement of the american heart association, and an expert consensus document of the American College of Cardiology Foundation. Diabetes Care. 2010;33(6):1395-402.

14. Ogawa H, Nakayama M, Morimoto T, Uemura S, Kanauchi M, Doi N, Jinnouchi H, Sugiyama S, Saito Y. Low-dose aspirin for primary prevention 
of atherosclerotic events in patients with type 2 diabetes: a randomized controlled trial. JAMA. 2008;300(18):2134-41.

15. Ikeda Y, Shimada K, Teramoto T, Uchiyama S, Yamazaki T, Oikawa S, Sugawara M, Ando K, Murata M, Yokoyama K, et al. Low-dose aspirin for primary prevention of cardiovascular events in Japanese patients 60 years or older with atherosclerotic risk factors: a randomized clinical trial. JAMA. 2014;312(23):2510-20.

16. Bowman L, Mafham M, Wallendszus K, Stevens W, Buck G, Barton J, Murphy K, Aung T, Haynes R, Cox J, et al. Effects of aspirin for primary prevention in persons with diabetes mellitus. N Engl J Med. 2018;379(16):1529-39.

17. Wallentin L, James S, Storey RF, Armstrong M, Barratt BJ, Horrow J, Husted S, Katus H, Steg PG, Shah SH, et al. Effect of CYP2C19 and ABCB1 single nucleotide polymorphisms on outcomes of treatment with ticagrelor versus clopidogrel for acute coronary syndromes: a genetic substudy of the PLATO trial. Lancet. 2010;376:1320-8.

18. Mega JL, Close SL, Wiviott SD, Shen L, Walker JR, Simon T, Antman EM, Braunwald E, Sabatine MS. Genetic variants in ABCB1 and CYP2C19 and cardiovascular outcomes after treatment with clopidogrel and prasugrel in the TRITON-TIMI 38 trial: a pharmacogenetic analysis. Lancet. 2010;376:1312-9.

19. Ajjan RA, Standeven KF, Khanbhai M, Phoenix F, Gersh KC, Weisel JW, Kearney MT, Ariens RA, Grant PJ. Effects of aspirin on clot structure and fibrinolysis using a novel in vitro cellular system. Arterioscler Thromb Vasc Biol. 2009;29(5):712-7.

20. Thomas MR, Outteridge SN, Ajjan RA, Phoenix F, Sangha GK, Faulkner RE, Ecob R, Judge HM, Khan H, West LE, et al. Platelet P2Y12 inhibitors reduce systemic inflammation and its prothrombotic effects in an experimental human model. Arterioscler Thromb Vasc Biol. 2015;35(12):2562-70.

21. Kiers $D$, van der Heijden WA, van Ede L, Gerretsen J, de Mast $Q$, van der Ven AJ, El Messaoudi S, Rongen GA, Gomes M, Kox M, et al. A randomised trial on the effect of anti-platelet therapy on the systemic inflammatory response in human endotoxaemia. Thromb Haemost. 2017;117(9):1798-807.

22. Morris T, Stables M, Hobbs A, de Souza P, Colville-Nash P, Warner T, Newson J, Bellingan G, Gilroy DW. Effects of low-dose aspirin on acute inflammatory responses in humans. J Immunol. 2009;183(3):2089-96.

23. Mayr M, Zampetaki A, Willeit P, Willeit J, Kiechl S. MicroRNAs within the continuum of postgenomics biomarker discovery. Arterioscler Thromb Vasc Biol. 2013;33(2):206-14.

24. Willeit P, Zampetaki A, Dudek K, Kaudewitz D, King A, Kirkby NS, CrosbyNwaobi R, Prokopi M, Drozdov I, Langley SR, et al. Circulating MicroRNAs as novel biomarkers for platelet activation. Circ Res. 2013;112(4):595-600.

25. Kaudewitz D, Skroblin P, Bender LH, Barwari T, Willeit P, Pechlaner R, Sunderland NP, Willeit K, Morton AC, Armstrong PC, et al. Association of MicroRNAs and YRNAs with platelet function. Circ Res. 2016;1 18:420-32.

26. Zampetaki A, Willeit P, Tilling L, Drozdov I, Prokopi M, Renard J-M, Mayr A, Weger S, Schett G, Shah A, et al. Prospective study on circulating MicroRNAs and risk of myocardial infarction. J Am Coll Cardiol. 2012;60(4):290-9.

27. Storey RF, Husted S, Harrington RA, Heptinstall S, Wilcox RG, Peters G, Wickens M, Emanuelsson H, Gurbel P, Grande P, et al. Inhibition of platelet aggregation by AZD6140, a reversible oral P2Y12 receptor antagonist, compared with clopidogrel in patients with acute coronary syndromes. J Am Coll Cardiol. 2007;50(19):1852-6.

28. Storey RF, Judge HM, Wilcox RG, Heptinstall S. Inhibition of ADPinduced P-selectin expression and platelet-leukocyte conjugate formation by clopidogrel and the P2Y12 receptor antagonist AR-C69931 MX but not aspirin. Thromb Haemost. 2002;88:488-94.

29. Neergaard-Petersen S, Ajjan R, Hvas AM, Hess K, Larsen SB, Kristensen $\mathrm{SD}$, Grove EL. Fibrin clot structure and platelet aggregation in patients with aspirin treatment failure. PLOS ONE. 2013;8:e71150.

30. Carter A, Cymbalista C, Spector T, Grant P. Heritability of clot formation, morphology, and lysis: the EuroCLOT study. Arterioscler Thromb Vasc Biol. 2007;27:2783-9.

31. Franchi F, Rollini F, Cho JR, King R, Phoenix F, Bhatti M, DeGroat C, Tello-Montoliu A, Zenni MM, Guzman LA, et al. Effects of dabigatran on the cellular and protein phase of coagulation in patients with coronary artery disease on dual antiplatelet therapy with aspirin and clopidogrel. Results from a prospective, randomised, double-blind, placebo-controlled study. Thromb Haemost. 2016;115(3):622-31.
32. Hess K, Alzahrani SH, Price JF, Strachan MW, Oxley N, King R, Gamlen T, Schroeder V, Baxter PD, Ajjan RA. Hypofibrinolysis in type 2 diabetes: the role of the inflammatory pathway and complement C3. Diabetologia. 2014;57(8):1737-41.

33. Neergaard-Petersen S, Hvas AM, Kristensen SD, Grove EL, Larsen SB, Phoenix F, Kurdee Z, Grant PJ, Ajjan RA. The influence of type 2 diabetes on fibrin clot properties in patients with coronary artery disease. Thromb Haemost. 2014;112(6):1142-50.

34. Al-Barjas HS, Ariens R, Grant P, Scott JA. Raised plasma fibrinogen concentration in patients with abdominal aortic aneurysm. Angiology. 2006;57(5):607-14.

35. Livak KJ, Schmittgen TD. Analysis of relative gene expression data using real-time quantitative PCR and the 2(-Delta Delta $C(T))$ Method. Methods. 2001;25(4):402-8.

36. Storey RF, Angiolillo DJ, Bonaca MP, Thomas MR, Judge HM, Rollini F, Franchi F, Ahsan AJ, Bhatt DL, Kuder JF, et al. Platelet Inhibition with Ticagrelor $60 \mathrm{mg}$ Compared with $90 \mathrm{mg}$ Twice-daily in the PEGASUSTIMI 54 study. J Am Coll Cardiol. 2016;67:1145-54.

37. Angiolillo DJ, Bernardo E, Sabaté M, Jimenez-Quevedo P, Costa MA, Palazuelos J, Hernández-Antolin R, Moreno R, Escaned J, Alfonso F, et al. Impact of platelet reactivity on cardiovascular outcomes in patients with type 2 diabetes mellitus and coronary artery disease. J Am Coll Cardiol. 2007;50(16):1541-7.

38. Bonello L, Tantry US, Marcucci R, Blindt R, Angiolillo DJ, Becker R, Bhatt DL, Cattaneo M, Collet JP, Cuisset T, et al. Consensus and future directions on the definition of high on-treatment platelet reactivity to adenosine diphosphate. J Am Coll Cardiol. 2010;56(12):919-33.

39. Zampetaki A, Kiechl S, Drozdov I, Willeit P, Mayr U, Prokopi M, Mayr A, Weger S, Oberhollenzer F, Bonora E, et al. Plasma microRNA profiling reveals loss of endothelial MiR-126 and other MicroRNAs in type 2 diabetes. Circ Res. 2010;107(6):810-7.

40. Seidu S, Kunutsor SK, Sesso HD, Gaziano JM, Buring JE, Roncaglioni MC, Khunti K. Aspirin has potential benefits for primary prevention of cardiovascular outcomes in diabetes: updated literature-based and individual participant data meta-analyses of randomized controlled trials. Cardiovasc Diabetol. 2019;18(1):70.

41. American Diabetes Association. Cardiovascular Disease and Risk Management: Standards of Medical Care in Diabetes-2019. Diabetes Care. 2019;42(Suppl 1):S103-23.

42. Arnett DK, Blumenthal RS, Albert MA, Buroker AB, Goldberger ZD, Hahn EJ, Himmelfarb CD, Khera A, Lloyd-Jones D, McEvoy JW, et al. 2019 ACC/AHA Guideline on the Primary Prevention of Cardiovascular Disease: a Report of the American College of Cardiology/American Heart Association Task Force on Clinical Practice Guidelines. Circulation. 2019;140(11):e596-646.

43. Vernstrom L, Funck KL, Grove EL, Laugesen E, Baier JM, Hvas AM, Poulsen PL. Antiplatelet effect of aspirin during $24 \mathrm{~h}$ in patients with type 2 diabetes without cardiovascular disease. Thromb Res. 2018;161:1-6.

44. Joshi RR, Hossain R, Morton AC, Ecob R, Judge HM, Wales C, Walker JV, Karunakaran A, Storey RF. Evolving pattern of platelet P2Y12 inhibition in patients with acute coronary syndromes. Platelets. 2014;25(6):416-22.

45. Rosenson RS, Chen Q, Najera SD, Krishnan P, Lee ML, Cho DJ. Ticagrelor improves blood viscosity-dependent microcirculatory flow in patients with lower extremity arterial disease: the Hema-kinesis clinical trial. Cardiovasc Diabetol. 2019;18(1):77.

46. Schmucker J, Fach A, Mata Marin LA, RetzlaffT, Osteresch R, Kollhorst B, Hambrecht R, Pohlabeln $\mathrm{H}$, Wienbergen $\mathrm{H}$. Efficacy and safety of ticagrelor in comparison to clopidogrel in elderly patients with ST-segmentelevation myocardial infarctions. J Am Heart Assoc. 2019;8(18):e012530.

47. Otsuki H, Yamaguchi J, Kawamoto T, Yoshikawa M, Ebihara S, Tanaka K, Nakao M, Jujo K, Arashi H, Ota Y, et al. Safety and efficacy of low-dose prasugrel as part of triple therapy with aspirin and oral anticoagulants in patients with atrial fibrillation undergoing percutaneous coronary intervention-from the TWMU-AF PCI registry. Circ J. 2019;83(5):1000-5.

48. Gurbel PA, Erlinge D, Ohman EM, Neely B, Neely M, Goodman SG, Huber K, Chan MY, Cornel JH, Brown E, et al. Platelet function during extended prasugrel and clopidogrel therapy for patients with ACS treated without revascularization: the TRILOGY ACS platelet function substudy. JAMA. 2012;308(17):1785-94. 
49. Brandt JT, Payne CD, Wiviott SD, Weerakkody G, Farid NA, Small DS, Jakubowski JA, Naganuma H, Winters KJ. A comparison of prasugre and clopidogrel loading doses on platelet function: magnitude of platelet inhibition is related to active metabolite formation. Am Heart J. 2007;153(1):66.e69.

50. Kondkar AA, Bray MS, Leal SM, Nagalla S, Liu DJ, Jin Y, Dong JF, Ren Q, Whiteheart SW, Shaw C, et al. VAMP8/endobrevin is overexpressed in hyperreactive human platelets: suggested role for platelet microRNA. J Thromb Haemost. 2010;8(2):369-78.
51. Schulte C, Molz S, Appelbaum S, Karakas M, Ojeda F, Lau DM, Hartmann T, Lackner KJ, Westermann D, Schnabel RB, et al. miRNA-197 and miRNA223 predict cardiovascular death in a cohort of patients with symptomatic coronary artery disease. PLoS ONE. 2015;10(12):e0145930.

\section{Publisher's Note}

Springer Nature remains neutral with regard to jurisdictional claims in published maps and institutional affiliations.
Ready to submit your research? Choose BMC and benefit from:

- fast, convenient online submission

- thorough peer review by experienced researchers in your field

- rapid publication on acceptance

- support for research data, including large and complex data types

- gold Open Access which fosters wider collaboration and increased citations

- maximum visibility for your research: over $100 \mathrm{M}$ website views per year

At BMC, research is always in progress.

Learn more biomedcentral.com/submissions 